\title{
A Numerical Comparison of Integral Equations of the First and Second Kind for Conformal Mapping
}

\author{
By John K. Hayes, David K. Kahaner and Richard G. Kellner*
}

\begin{abstract}
Two methods for computing numerical conformal mappings are compared. The first, due to Symm, uses a Fredholm integral equation of the first kind while the other, due to Lichtenstein, uses a Fredholm integral equation of the second kind. The two methods are tested on ellipses with different ratios of major to minor axes. The method based on the integral equation of the second kind is superior if the ratio is less than or equal to 2.5. The opposite is true if the ratio is greater than or equal to 10. Similar results are obtained for other regions.
\end{abstract}

Introduction. Let $D$ be a bounded simply-connected region in the plane with a smooth boundary curve $L$. Then it is well known that there is a conformal mapping $f$ which takes $D$ in a $1-1$ fashion onto the unit disc $|\zeta|<1$ in such a way that a given point $z_{0} \in D$ is carried into $f\left(z_{0}\right)=0$. Moreover, $f$ is uniquely determined up to an arbitrary rotation of the disc, and $f$ can be extended continuously to $D+L$. The determination of $f$ is equivalent to the determination of the Green's function of $D$.

Recently, Symm [12] suggested a method for conformal mapping which represents the regular part of the Green's function of $D$ as a single-layer potential on $L$. This results in a Fredholm integral equation of the first kind with a logarithmic kernel. An algorithm based on this integral equation for computing the mapping numerically [12], as well as an improved version [5], compared favorably with numerical methods based upon orthogonal polynomials.

The utility of integral equations of the first kind for the solution of numerical problems has been viewed with skepticism. Indeed, a search of the literature shows that, whenever a problem can be formulated both as an equation of the first kind and as an equation of the second kind, the latter is almost always chosen. Recent work, however, shows that equations of the first kind can be the basis for useful numerical procedures (see [7], [4], [10]).

The feasibility of numerically solving the integral equation used in Symm's method depends upon the nature of the spectrum of the associated operator. In [11]

Received July 18, 1973

AMS (MOS) subject classifications (1970). Primary 30A28; Secondary 65E05.

Key words and phrases. Numerical conformal mapping, numerical solution of integral equations of the first kind.

*This research was supported by the U. S. Atomic Energy Commission under Contract W-7405-ENG-36. 
it is shown that the spectrum of the operator associated with Symm's method is real, and that the $n$th eigenvalue tends to zero like $n^{-1}$. Since this is relatively slow convergence to zero, we expect that a numerical method for solving the associated equation of the first kind should converge.

In this paper, we compare the numerical method of conformal mapping described in [5], which uses the integral equation of Symm, with a numerical method which uses an integral equation of the second kind. For the latter we have chosen the numerical procedure described in [1] which uses the integral equation of Lichtenstein. A description and comparison of various methods based upon integral equations of the second kind may be found in the comprehensive book of Gaier on conformal mapping [2].

Description of the Methods. Let $D, L, f(z)$, and $z_{0}$ be as before. We assume that $L$ has continuous curvature and that $f$ has been extended by continuity to $D+$ $L$. The assumption on $L$ is more restrictive than is necessary, but we shall not be concerned with weakening it.

(a) Symm's Method. It is known that $f(z)$ can be represented as

$$
f(z)=\left(z-z_{0}\right) \exp [g(z)+i h(z)]
$$

where $g$ and $h$ are real-valued harmonic conjugates, and $g$ satisfies the boundary-value problem

$$
\begin{aligned}
\nabla^{2} g(z) & =0, & z \in D, \\
g(z) & =-\log \left|z-z_{0}\right|, & z \in L .
\end{aligned}
$$

Moreover, $g(z)$ can be represented as a single-layer potential with continuous density function $\mu(\zeta)$,

$$
g(z)=\int_{L} \mu(\zeta) \log |z-\zeta||d \zeta|, \quad z \in D
$$

subject to a possible rescaling of $D$ (see [9]).

In the case that the transfinite diameter [6] of $D$ is precisely equal to 1 , a constant might have to be added to the above representation (2) for $g$. However, we do not concern ourselves with this case for the following reason. If the transfinite diameter of $D$ is 1 , then the operator $A$ defined by

$$
(A \varphi)(z)=\int_{L} \varphi(\zeta) \log |z-\zeta||d \zeta|, \quad z \in L,
$$

has a zero eigenvalue [6]. This would lead to nonuniqueness and an ill-conditioned system of equations in the numerical scheme. Thus we assume, by means of a preliminary rescaling of $D$ if necessary, that the transfinite diameter of $D$ is not 1 .

Since the single-layer potential in (2) is continuous everywhere, including the 
curve $L$, the representation (2) along with the boundary values for $g(z)$ lead to the equation

$$
\int_{L} \mu(\zeta) \log |z-\zeta||d \zeta|=-\log \left|z-z_{0}\right|, \quad z \in L
$$

After (3) has been solved for $\mu(\zeta)$, the mapping problem has essentially been solved. For then $g$ and $h$ are obtained by the quadratures

$$
\begin{aligned}
& g(z)=\int_{L} \mu(\zeta) \log |z-\zeta||d \zeta|, \\
& h(z)=\int_{L} \mu(\zeta) \arg (z-\zeta)|d \zeta|
\end{aligned}
$$

for any $z \in D+L$, and $f(z)$ is then determined by (1). The function $h(z)$ is only determined to within an arbitrary constant, and this corresponds to an arbitrary rotation of the unit disc.

The corresponding numerical procedure consists of discretizing equation (3), solving it for a discrete approximation to $\mu(\zeta)$, and finally performing numerical quadratures to obtain approximations to $g, h$, and thus $f$. A complete description of the numerical procedure which we used for the following tests may be found in [5]. We denote this method by (S).

(b) Lichtenstein's Method. Take $z_{0}=0$ for convenience. Consider the singlevalued analytic function in $D$

$$
w(z)=i \log [z / f(z)]
$$

and let $w=u+i v$, where $u, v$ are real-valued functions. The function $u(z)=$ $\arg (f(z))-\arg (z), z \in L$, is the angular distortion function.

By applying Green's third identity to $u(z)$, Lichtenstein [8] obtained the integral equation

$$
u(z)=\frac{1}{\pi} \int_{L} N(z, \zeta) u(\zeta)|d \zeta|+\Phi(z), \quad z \in L
$$

where

$$
N(z, \zeta)=\partial(\log |z-\zeta|) / \partial n_{\zeta}
$$

and

$$
\Phi(z)=\frac{1}{\pi} \int_{L} \log |\zeta| d \log |z-\zeta|
$$

Here, $n_{\zeta}$ is the unit outer normal to $L$ at the point $\zeta \in L$, and the integral in (8) is taken in the sense of the Cauchy principal value. $N(z, \zeta)$ is the Neumann kernel.

As was the case with (S), the conformal mapping may be easily found once the integral equation has been solved. For if (7) has been solved for $u(z)$, then $f(z)$ may be found for all $z \in L$ by (6), since by definition $v(z)$ is $\log |z|$ on the boundary curve 
$L$. The Cauchy formula for analytic functions then completely determines $f(z)$ in $D$.

We use the numerical implementation of the above which was described in [1] and which we summarize here. The integral in (8) is singular, so the alternate representation

$$
\Phi(z)=\frac{1}{\pi} \int_{L}[\arg (\zeta)-2 \arg (\zeta-z)] d \arg (\zeta-z)+\arg (z)+\pi
$$

is used. The integrand in (9) is a smooth periodic function, and the trapezoidal rule, which in a sense is the most accurate quadrature formula for periodic functions [1], is used to evaluate (9). The right-hand side of (7) is discretized by means of the trapezoidal rule, and the resulting linear algebraic equations are solved by successive substitution. We shall denote this method by (BYZ/L).

Solutions to (7) are determined only to within an arbitrary constant, since

$$
\frac{1}{\pi} \int_{L} N(z, \zeta)|d \zeta|=1
$$

This corresponds to the fact that $f(z)$ is determined only to within an arbitrary rotation of the unit disc. Thus it is convenient to use the pseudonorm

$$
\|u\|=\sup _{z_{j}, z_{k} \in L}\left|u\left(z_{j}\right)-u\left(z_{k}\right)\right|
$$

which identifies functions differing by a constant. It is well known that the Picard iterates of (7) converge geometrically in this pseudonorm for any $L$ and any function $\Phi$. A simple proof of this is given in [1] for convex regions.

The iterates of the discretization of (7) need not converge. This does not seem to have been observed in the literature. Indeed, in [1] it is stated, referring to the aforementioned convergence proof, that "the preceding argument has the advantage of applying also to the discretization... ." This assumes, however, that the value of the discretization of $(1 / \pi) \int_{L} N(z, \zeta)|d \zeta|$ is approximately equal to 1 for all mesh points $z \in L$. We have observed, numerically, that values somewhat greater than 1 (say 1.1) still yield convergent iterations. For the $10: 1$ ellipse given below, the approximation to the integral using a coarse mesh was greater than 2 for some $z$ and the iteration diverged. In this case, we solved the discretized equations by Gaussian elimination. The answers were not even correct in the first digit.

Testing. Calculations with (S) were performed using the program described in [5] . (BYZ/L) was coded according to the description in [1], which was summarized above. As a benchmark, and to provide confidence in the validity of our version of this code, we attempted to duplicate the results in [1, Table I]. Our answers were found to be about 5-10\% more accurate, presumably because of word-length differences. ${ }^{* *}$

${ }^{* *}$ This allows for the fact that the "exact" values given in [1] are slightly in error. 
For our test problems, we chose the regions bounded by the curves

$$
(x / \rho)^{2}+y^{2}=1, \quad \rho=1.25,2.5,5.0,10.0,20.0,
$$

$$
\begin{aligned}
& x=[2+\epsilon \sin (k \theta)] \cos \theta \\
& y=[2+\epsilon \sin (k \theta)] \sin \theta, \quad 0 \leqslant \theta \leqslant 2 \pi, \quad k=3,7, \quad \epsilon=.1, .25, .5, .75,1.0 .
\end{aligned}
$$

Thus, there are fifteen different geometries. In each case, the origin was mapped into itself and no attempt was made to capitalize on the symmetry of the region. The problems above were run by discretizing the boundary into $32,64,128,256$, and 512 segments of equal arc length. The arc length was computed to within only $10^{-9}$, which limits the numerical mapping to this accuracy. Thus, there were a total of 75 cases. For each geometry, we use the results for the 512-point case as a standard. A comparison of these cases from (BYZ/L) and (S) showed sufficient agreement to justify this approach. Furthermore, the conformal mapping of an ellipse may be found analytically. Numbers calculated from the analytic expression also confirm that the above approach is valid.

Because of the maximum modulus principle, we only compare errors on the boundary. Moreover, we limit ourselves to comparing errors in argument, since it has been our experience with (S) that errors in argument are about equal to errors in modulus. We summarize our results in a series of graphs, one for each geometry. On each graph we plot for $(\mathrm{BYZ} / \mathrm{L})$ and $(\mathrm{S})$ a measure of the error versus the number of boundary points used in the approximation. The measure of error which we use is the error in argument (relative to the 512-point standards) averaged over the 32 points common to all calculations for the geometry in question. (BYZ/L) did not converge for some problems. The results for these cases are not plotted.

Test Results, Modifications, and Programming Problems. If the domain is badly distorted, (BYZ/L) may fail to converge while (S) still provides acceptable answers. For almost circular domains, (BYZ/L) is both accurate and converges in only a few iterations. Regions of average distortion are mapped about equally well when both methods use small numbers of boundary points. Note that, since the boundary curves for the test regions are analytic, the accuracy in $(\mathrm{BYZ} / \mathrm{L})$ should improve superlinearly because trapezoidal quadrature is used. In some cases, this superlinear convergence is apparent. In others, the accelerated convergence is only beginning to appear with 256 points. The amount of computer time for (BYZ/L) varies with the number of iterations necessary for convergence, but, for the 75 problems tested, the total time for (BYZ/L) was amost $2 / 3$ that of (S), allowing a maximum of 50 iterations. Inspection showed that those calculations requiring all 50 iterations were, in fact, diverging. Memory requirements are about equal for (BYZ/L) and (S) since both need to store an $N \times N$ matrix, where $N$ is the number of boundary points used in the approximation. 
Table Ia

Conformal map of $\left(\frac{x}{\rho}\right)^{2}+y^{2}=1$.

Average error vs. number of boundary points.
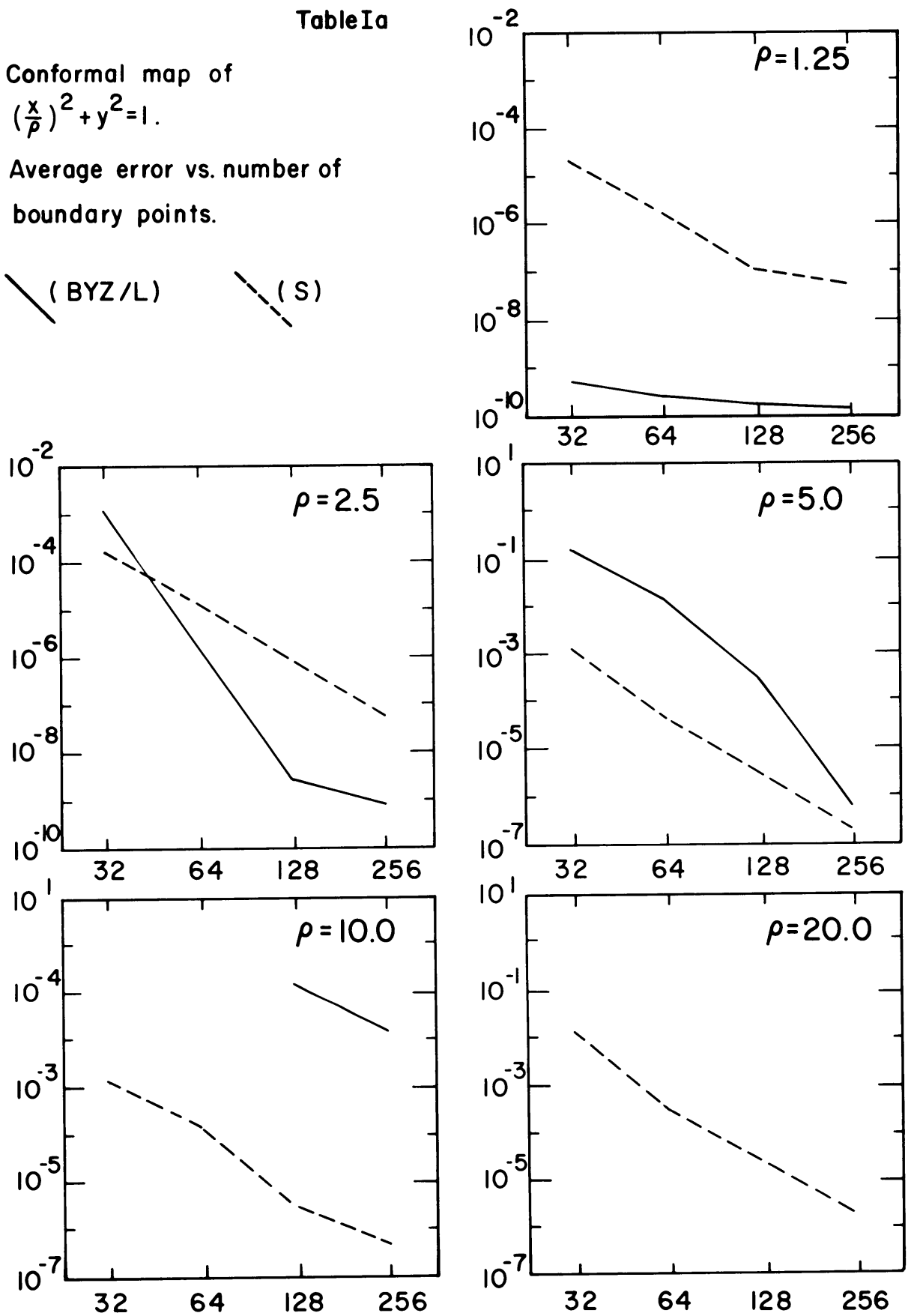

The algorithm used by (S) allows for boundary points that are not equally spaced with respect to arc length, and (BYZ/L) can also be so modified. This has been shown in [5] and [1] to enable one to obtain greatly improved accuracy with the same total number of boundary points. However, the optimum spacing of boundary points for 


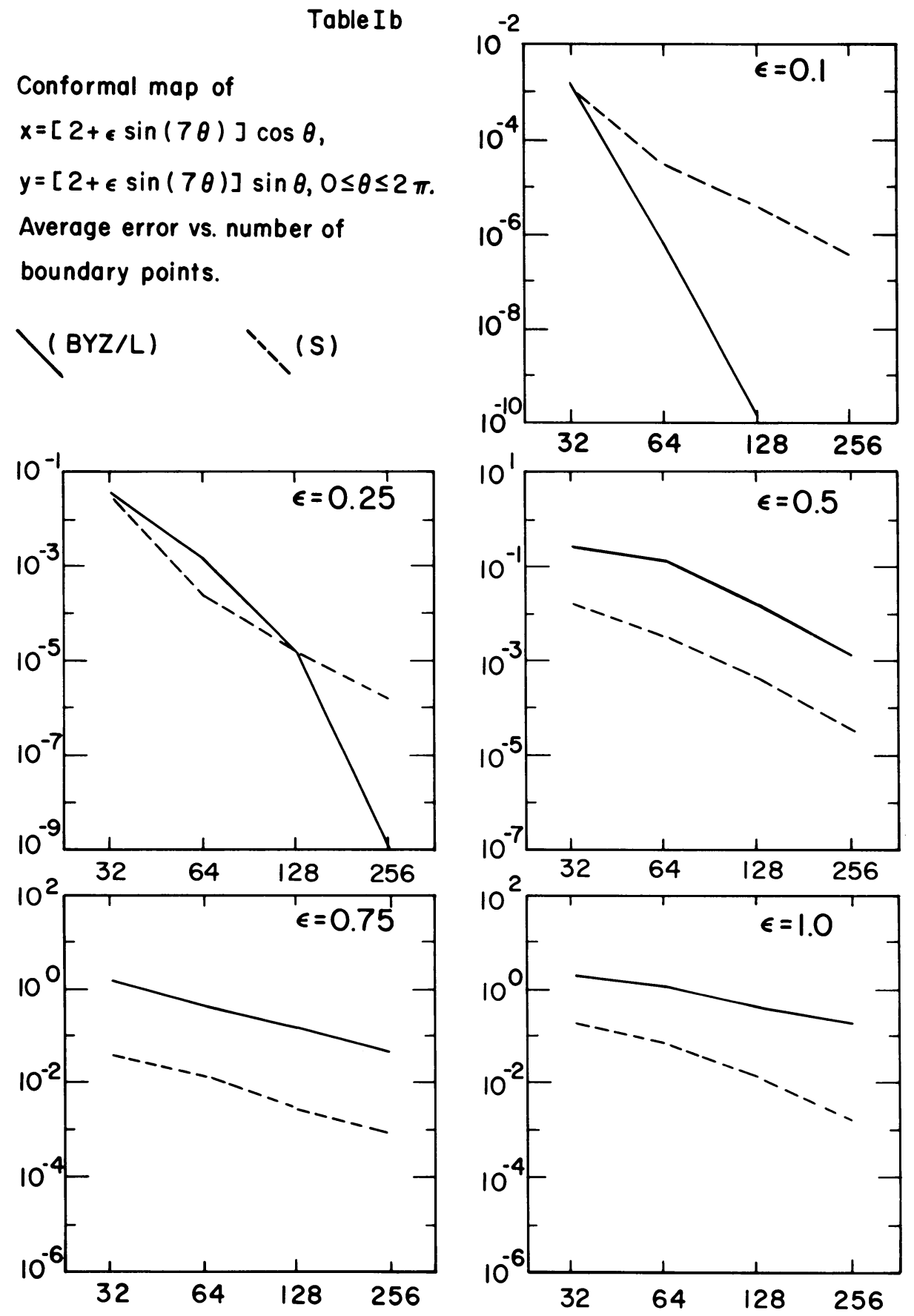

(BYZ/L) is not consistent with the optimum spacing for (S) because of the different kernels. So as not to penalize either algorithm in our tests, we distributed points equally with respect to arc length.

In [1], the authors suggest computing $\Phi(z)$ by the trapezoidal rule with $N$ points. 
Table Ic

\section{Conformal map of}

$x=[2+\epsilon \sin (3 \theta)] \cos (\theta)$,

$y=[2+\epsilon \sin (3 \theta)] \sin (\theta), 0 \leq \theta \leq 2 \pi$.

Average error vs. number of boundary points.
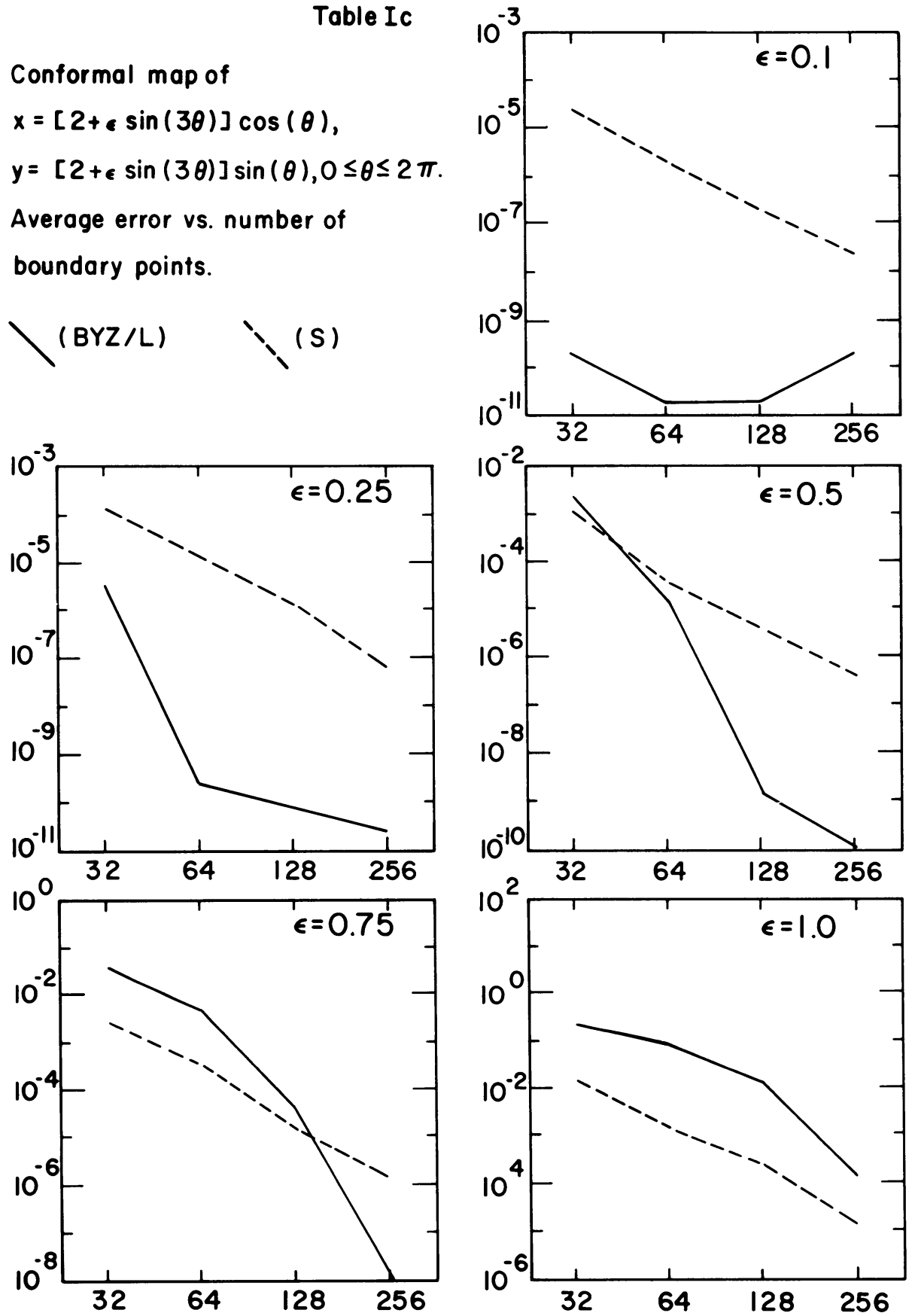

However, the integrand in $\Phi(z)$ can be quite bad. For example, with $\rho=10$ in (a) above, the integrand looks like an approximation to the derivative of a delta function. Therefore, there seemed to us to be no reason to tie the evaluation of this integral to the discretization in the integral equation. Consequently, we repeated some of the calculations in $(\mathrm{BYZ} / \mathrm{L})$ using the trapezoidal rule with more points to compute $\Phi$, 
Table II

Conformal map of $\left(\frac{x}{\rho}\right)^{2}+y^{2}=1$

Average error vs. number of boundary points $N$. $\phi_{m}$ indicates that $\mathrm{m} N$ boundary points were used in the computation of $\phi$.
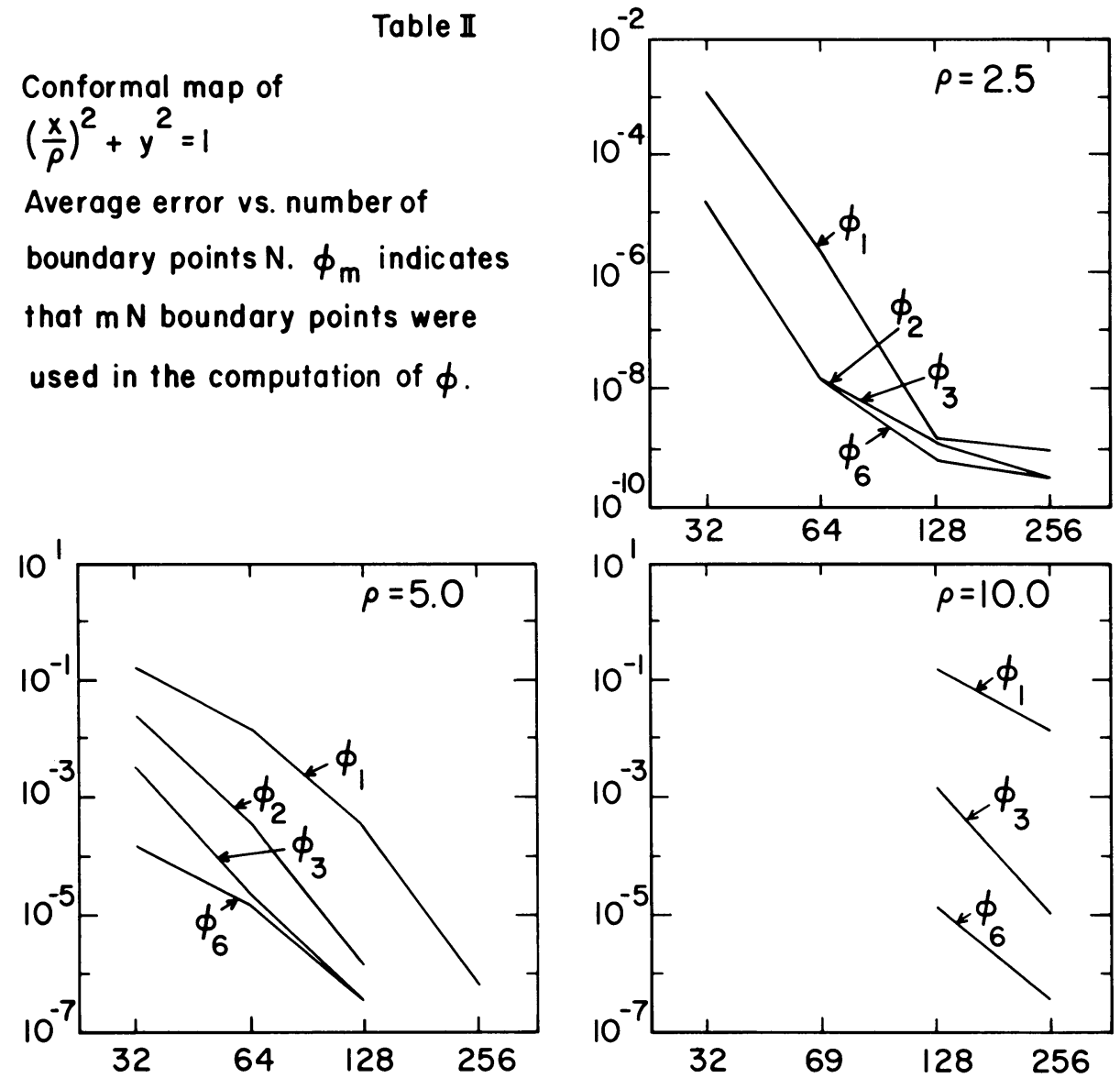

while keeping $N$ equations. The results are shown in the preceding graphs. The errors never got any worse and, in most cases, they were substantially smaller. The trapezoidal approximation to $\Phi$ requires $O\left(N^{2}\right)$ calculations. Additional storage and time costs are minimal compared to increasing the size of the matrix.

We feel that (BYZ/L) was a good representative of the various methods of conformal mapping using integral equations of the second kind with which to have compared (S). We also tested the methods of Gershgorin and of Carrier, which use equations of the second kind and which were discussed in [1]. We feel, in agreement with [1], that (BYZ/L) has certain advantages over these methods. A fairly complete summary of the results obtained by using these various methods may be found in [2] . We note that, although the method of Carrier was stated correctly in Eqs. (11) and $\left(11^{\prime}\right)$ of [1], it was, unfortunately, stated incorrectly on p. 119 of [1] and this has been carried over to the book of Gaier [2].

Conclusions. Our tests have shown that the integral equation of the first kind can be a useful tool as a basis for numerical procedures, and that results obtained with it can compete with those obtained using equations of the second kind. 
The method (BYZ/L) is clearly better than (S) if the domain to be mapped is not excessively distorted from a circle. Even if the domain is distorted, (BYZ/L) may be better if the mesh is sufficiently fine and special care is used in computing the function $\Phi(z)$.

The method (S), on the other hand, will handle very distorted domains, even with a relatively small number of boundary points. Furthermore, although it has not been stated above, our tests have shown that (S) more often gave a discrete approximation to $\arg (f(z(s)))$ which was a monotone increasing function of $s$. Clearly, this function has this property.

If the boundary of the region to be mapped has a corner, the equations must be altered at the corner with (BYZ/L) but not with (S). Furthermore, the superlinear convergence of the trapezoidal rule is lost. Finally, if the image of an interior point is required, an additional procedure is needed for $(\mathrm{BYZ} / \mathrm{L})$.

Acknowledgment. We would like to thank Professor Garrett Birkhoff for his suggestions and comments concerning this paper.

University of California

Los Alamos Scientific Laboratory

Los Alamos, New Mexico 87544

1. G. BIRKHOFF, D. M. YOUNG \& E. H. ZARANTONELLO, Numerical Methods in Conformal Mapping, Proc. Sympos. Appl. Math., vol. IV, McGraw-Hill, New York, 1953, pp. 117-140. MR 15, 258.

2. D. GAIER, Konstruktive Methoden der konformen Abbildung, Springer-Verlag, Berlin, 1964. MR 33 \#7507.

3. C. GRAM (Editor), Selected Numerical Methods for Linear Equations, Polynomial Equations, Partial Differential Equations, Conformal Mappings, Regnecentralen, Copenhagen, 1962. MR 26 \#7423.

4. J. HAYES, Four Computer Programs Using Green's Third Formula to Numerically Solve Laplace's Equation in Inhomogeneous Media, Los Alamos Scientific Laboratory Report, LA-4423 (April 1970).

5. J. K. HAYES, D. K. KAHANER \& R. KELLNER, "An improved method for numerical conformal mapping," Math. Comp., v. 26, 1972, pp. 327-334. MR 46 \#334.

6. J. K. HAYES AND R. KELLNER, "The eigenvalue problem for a pair of coupled integral equations arising in the numerical solution of Laplace's equation," SIAM J. Appl. Math., v. 22, 1972, pp. 503-513. MR 46 \#4764.

7. M. A. JASWON, "Integral equation methods in potential theory. I," Proc. Roy. Soc. Ser. $A$, v. 275, 1963 , pp. 23-32. MR 27 \#4034.

8. L. LICHTENSTEIN, "Zur konformen Abbildung einfach zusammenhängender schlichter Gebiete," Arch. Math. Phys., v. 25, 1917, pp. 179-180.

9. N. I. MUSHELIŠVILI, Singular Integral Equations. Boundary Problems of Function Theory and Their Application to Mathematical Physics, Noordhoff, Groningen, 1953. MR 15, 434.

10. B. NOBLE, Conference on Applications of Numerical Analysis, (J. Morris, Editor), Springer-Verlag, Berlin, 1971.

11. S. SHAMMA, "A generalization of separability in boundary value problems," SIAM $J$. Appl. Math., v. 20, 1971 , pp. 536-546.

12. G. T. SYMM, "An integral equation method in conformal mapping," Numer. Math., v. 9, 1966, pp. 250-258. MR $34 \# 7056$.

13. S. E. WARSCHAWSKI, Recent Results in Numerical Methods of Conformal Mapping, Proc. Sympos. Appl. Math., vol. VI, Amer. Math. Soc., Providence, R. I., 1956, pp. 219-250. MR 19, 180. 